\title{
Microbiological profile of biliary tract infections
}

\author{
Deepinder Kaur ${ }^{1}$, Veenu Gupta', Rajoo Singh Chhina ${ }^{2}$, Daaman Sharma', \\ Charu Arora', Rama Gupta ${ }^{1}$ \\ ${ }^{1}$ Department of Microbiology, ${ }^{2}$ Department of Gastroenterology, \\ Dayanand Medical College \& Hospital, Ludhiana, Punjab, India.
}

\begin{abstract}
Background and objectives: Bacterial infection of biliary tract may cause severe inflammatory response or sepsis. An immediate bile culture and appropriate antibiotic administration are important to control the biliary tract infection. The objective of the study was to study the microbial profile and antibiotic sensitivity pattern in patients with biliary tract infection. Materials and methods: Fifty suspected cases of biliary tract infection admitted to the Department of Gastroenterology were enrolled. Bile samples from these patients were aseptically collected and sent to the Department of Microbiology. Samples were processed in automated BACTEC or BacT/ALERT system. Further identification and antimicrobial susceptibility testing was done by VITEK-2 system. Results: Of the 50 suspected cases of biliary tract infection, the majority were male patients and in the age group of 51-60 years. Growth was obtained in 22 (44\%) bile samples. The organisms obtained were Escherichia coli (40\%), Klebsiella spp. (20\%), Pseudomonas spp. (16\%), and coagulase-negative Staphylococci (8\%). All Gram negative isolates were susceptible to tigecycline and colistin. A high susceptibility was seen to amikacin and carbapenems while low susceptibility was seen to others. All the Gram positive organisms were sensitive to vancomycin, teicoplanin and linezolid with low sensitivity to penicillin. Interpretation and conclusion: $E$. coli is the most common organism isolated from bile. Antimicrobial sensitivity patterns require a revision of empiric antibiotic therapy policy in cholangitis. Early detection and determination of antimicrobial susceptibility pattern is important to reduce the mortality and morbidity associated with bile fluid infections.
\end{abstract}

Keywords: Antimicrobial susceptibility, bile culture, Gram negative bacteria

\section{INTRODUCTION}

The bile is normally sterile but in the presence of obstruction, the chances of cholangitis increases. ${ }^{[1,2]}$ Acute cholangitis refers to inflammation of the biliary ductal system from bacterial or non-bacterial infection, usually in the setting of biliary obstruction. ${ }^{[3]}$ However, the presence of gallstones within either the gallbladder or biliary tree is associated with the bacterial colonization of the bile. ${ }^{[4]}$ In patients without stone disease, previous biliary intervention is associated with

Corresponding Author: Prof. Deepinder Chhina

E-mail: deepinder.chhina@rediffmail.com

Received: 29-07-2015

Accepted: 24-09-2015

How to cite this article: Kaur D, Gupta V, Chhina RS, Sharma D, Arora C, Gupta R. Microbiological profile of biliary tract infections. J Gastrointest Infect, 2015; 5: 20-23. high rates of bacteriobilia. ${ }^{[5,6]}$ Under conditions of normal bile flow, bacteria in the biliary system are of no clinical significance. Upon bile duct obstruction, bacteria proliferate within the stagnant bile while biliary pressure increases. Eventually, the bacteria presumably translocate into the circulation causing a systemic infection. Acute cholangitis spans a continuous clinical spectrum and can progress from a local biliary infection to advanced disease with sepsis and multiple organ dysfunction syndrome. ${ }^{[7]}$ Even recent studies have reported mortality rates of up to $10 \% .{ }^{[8,9]}$

The most common organisms isolated in bile are Escherichia coli, Klebsiella spp., Enterococcus spp., Streptococcus spp., Enterobacter spp., Pseudomonas spp. and Candida spp. ${ }^{[1,2,3]}$ Microbiology of cholangitis has not changed significantly but the emergence of drug resistance among these organisms has been a matter of 
concern. Although definitive management of cholangitis involves the relief of bile stasis, effective empiric antibiotic therapy is an indispensable part of the treatment. ${ }^{[7,10]}$ Antimicrobial therapy recommendations state that antibiotics should be administered as soon as the diagnosis of acute cholangitis is suspected or established. Therefore, the present study was undertaken to know the microbiological profile and current antibiotic susceptibility patterns of isolates from patients with biliary tract infection admitted in a teaching hospital in North India.

\section{MATERIALS AND METHODS}

Fifty suspected cases of biliary tract infection admitted to the Department of Gastroenterology were enrolled. Bile samples from these patients were aseptically collected and sent to the Department of Microbiology. Samples were processed in automated BACTEC or BacT/ALERT system. Smears were prepared from the positive culture bottles and Gram staining of the smears was done. Simultaneously all the positive bottles were sub-cultured on blood agar and MacConkey agar plates. The plates were incubated at $37^{\circ} \mathrm{C}$ for $18-24$ hours. Identification of isolated organisms and antimicrobial susceptibility was done by VITEK-2 system. ${ }^{[11]}$

\section{RESULTS}

Among 50 cases suspected with biliary tract infection, $56 \%$ were males. Mean age of the patients was 55.2 years. Of the 50 bile samples, growth was obtained in $22(44 \%)$ samples. Bile culture positivity was more in females (50\%) as compared to males (40\%). Amongst the culture positive cases, maximum number of females and males were in the age group 61-70 years and 51-60 years respectively. The mean age of patients with positive bile culture was 54.6 years. Monomicrobial infection was seen in $19(86 \%)$ cases whereas polymicrobial was seen in $3(14 \%)$ culture positive cases. A total of 25 isolates were obtained from 22 samples. Majority of the isolates were Gram negative (84\%), followed by Gram positive $(12 \%)$ and yeasts $(4 \%)$. Among the isolates the most common one was E. coli (40\%), followed by Klebsiella pneumoniae (20\%), Pseudomonas aeruginosa (16\%) and coagulase-negative Staphylococci (8\%) (Fig.1). All the Gram negative isolates were susceptible to tigecycline and colistin. They also showed high susceptibility to

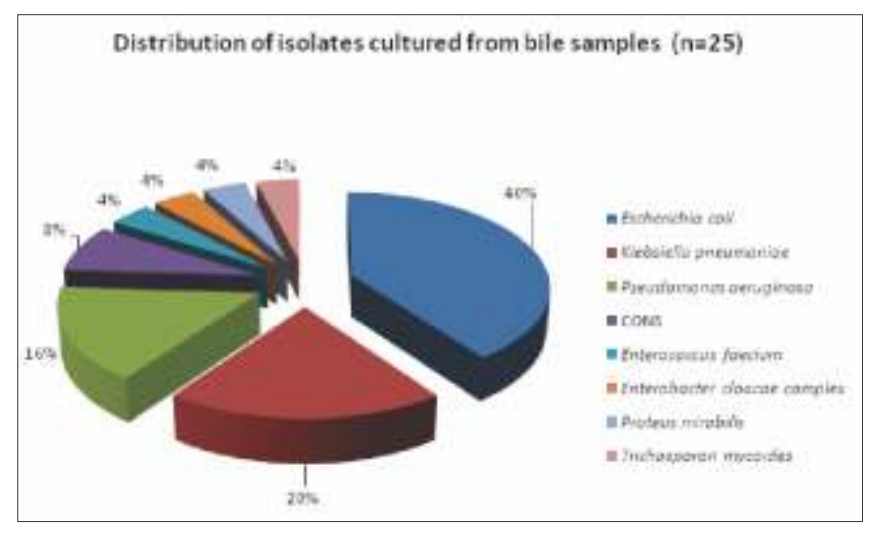

Fig. 1: Distribution of isolates cultured from bile samples $(n=25)$

amikacin (71.4\%) and carbapenems (76.2\%) while low susceptibility was seen to cefuroxime (20\%), fluoroquinolones $(23.8 \%)$, piperacillin/tazobactam (28.6\%) and cefoperazone/sulbactam (28.6\%) (Fig.2). Low susceptibility was seen for cephalosporins $(20 \%$ each) and ciprofloxacin (20-30\%) by K. pneumoniae and E. coli. High susceptibility was seen to aminoglycosides and carbapenems. P. aeruginosa had no sensitivity to ceftazidime, low sensitivity to fluoroquinolones (20$50 \%$ ) and carbapenems (20-50\%). All the Gram positive organisms were resistant to penicillin, whereas they showed $100 \%$ susceptibility to vancomycin, teicoplanin and linezolid. A single fungal isolate was identified as Trichosporon mycoides and was sensitive to fluconazole, voriconazole, flucytosine, amphotericin B and

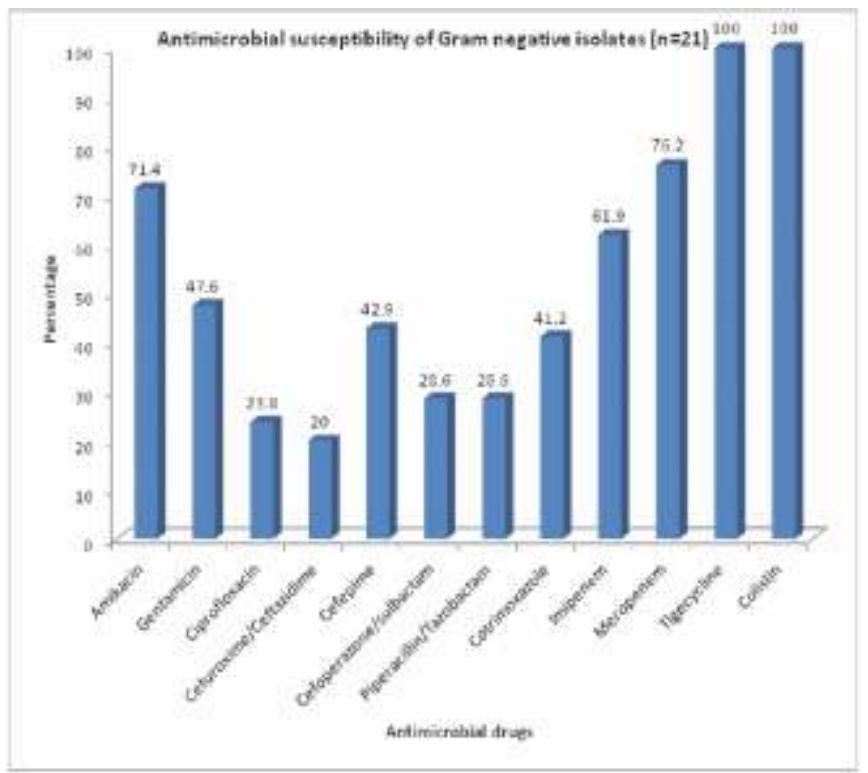

Fig.2: Antimicrobial susceptibility of Gram negative isolates $(n=21)$ 
capsofungin drugs.

\section{DISCUSSION}

Infected bile in cholangitis is a pivotal risk factor in increasing rate of complications such as bacteremia, wound infection and intra-abdominal sepsis. Majority of the patients were in the age group of 51-60 years and were males. In our study, bile culture positivity was $44 \%$. Our results are comparable with results of study by Fan et $a l^{[12]}$ and Shenoy et al ${ }_{1}^{[13]}$ who showed $23-46 \%$ and $56 \%$ culture positive rates respectively. ${ }^{[12,13]}$ Bile culture positivity was more in females as compared to males. Amongst the culture positive cases, maximum number of females and males were in the age group 61-70 years and 51-60 years respectively. In a study done in UK, maximum patients with positive bile cultures had mean age of $63.8 \pm 9.7$ years. ${ }^{[14]}$

Monomicrobial growth was obtained in 19 (86\%) bile samples. Similar finding was reported by Kaya et al ${ }^{[15]}$ in which $95 \%$ patients had monomicrobial growth in bile culture. The Gram negative enteric organisms formed $84 \%$ of the isolates. E. coli $(40 \%)$ was the most common isolate, followed by Klebsiella pneumoniae (20\%) and $P$. aeruginosa (16\%). Similar findings were seen in a study by Suri et al ${ }^{[16]}$ and Bae et al, ${ }^{[17]}$ in which E. coli was the most common isolate. ${ }^{[16,17]}$ In a study, Gram negative enteric aerobes accounted for $70-78 \%$ of positive bile cultures, in which E. coli and Klebsiella spp were the most common organisms. Vaishnavi et al ${ }^{[18]}$ investigated bile samples from 445 patients with biliary diseases as well as those requiring biliary drainage for other miscellaneous gastrointestinal diseases with special emphasis on Salmonellae. Bactericholia was detected in $68.8 \%$ patients with E.coli (17.5\%) followed by $K$. pneumoniae $(15.7 \%)$ and P. aeruginosa $(14.6 \%)$ as the most common microorganisms isolated. Salmonellae were also isolated from $5.8 \%$ of the samples. However they did not study the antibiotic sensitivity profile of these organisms.

Among Gram negative isolates, $100 \%$ were sensitive to tigecycline and colistin. They also showed high susceptibility to amikacin and carbapenems, while low susceptibility was seen to cephalosporins, fluoroquinolones, piperacillin/tazobactam and cefoperazone/ sulbactam. High sensitivity to amikacin (86\%), carbapenems (83\%) and piperacillin/ tazobactam (61\%), while low susceptibility to ampicillin (21\%) and cefotaxime $(14 \%)$ was observed by Kaya et al. ${ }^{[15]}$ Coagulase negative Staphylococci (CONS) accounted for $8 \%$ of the total isolates, which was in concordance with Bae et al ${ }^{[17]}$ who reported CONS to be $9.7 \%$ of the isolates. $^{[17]}$ All the Gram positive organisms were sensitive to vancomycin, teicoplanin and linezolid. Similar findings were seen in a study in Korea. ${ }^{[17]}$

To conclude, in the present study Gram negative bacteria were the commonest isolates from bile and showed high resistance to fluoroquinolones and cephalosporins. Thus, knowledge of the common etiologic agents and their local susceptibility profile is essential to ensure the appropriate choice of empiric antimicrobial therapy.

\section{Conflict of interest: Nil}

\section{REFERENCES}

1. Yusoff IF, Barkun JS, Barkun AN. Diagnosis and management of cholecystitis and cholangitis. Gastroenterol Clin North Am. 2003;32:1145-68

2. Kadakia SC. Biliary tract emergencies Acute cholecystitis, acute cholangitis and acute pancreatitis. Med Clin North Am. 1993;77:1015-36.

3. Qureshi WA. Approach to the patient who has suspected acute bacterial cholangitis. Gastroenterol Clin North Am. 2006;35: 409-23.

4. Csendes A, Fernandez M, Uribe P. Bacteriology of the gallbladder bile in normal subjects. Am J Surg. 1975;129:629-31.

5. Hochwald SN, Burke EC, Jarnagin WR, Fong Y, Blumgart LH. Association of preoperative biliary stenting with increased postoperative infectious complications in proximal cholangiocarcinoma. Arch Surg. 1999;134:261-266.

6. Nomura T, Shirai Y, Hatakeyama K. Bacteribilia and cholangitis after percutaneous transhepatic biliary drainage for malignant biliary obstruction. Dig Dis Sci. 1999;44:542-6.

7. Tanaka A, Takada T, Kawarada Y, Nimura Y, Yoshida M, Miura F, et al. Antimicrobial therapy for acute cholangitis: Tokyo Guidelines. J Hepatobiliary Pancreat Surg. 2007; 14:59-67.

8. Lai EC, Mok FP, Tan ES, Lo CM, Lo CM, Fan ST, et al. Endoscopic biliary drainage for severe acute cholangitis. $\mathrm{N}$ Engl $\mathrm{J}$ Med. 1992;326:1582-6.

9. Leung JW, Chung SC, Sung JJ, Banez VP, Li AK. Urgent endoscopic drainage for acute suppurative cholangitis. Lancet. 1989;1:1307-9.

10. Salvador VB, Lozada MC, Consunji RJ. Microbiology and antibiotic susceptibility of organisms in bile cultures from patients with and without cholangitis at an Asian academic medical center. Surg Infect (Larchmt). 2011;12:105-11.

11. Winn JW, Washington W, Allen S, Janda W, Koneman E, Procop $\mathrm{G}$ et al. Introduction to Microbiology: Part II: Guidelines for the collection, transport, processing, analysis and reporting of cultures from specific specimen sources. In: Koneman's color atlas and Textbook of Diagnostic Microbiology. $6^{\text {th }}$ ed. Philadelphia: Lippincot Willams and Willikins; 2006.p.103-5.

12. Fan ST, Lai EC, Mok FP, Choi TK, Wong J. Acute cholangitis secondary to hepatolithiasis. Arch Surg. 1991;126:1027-31. 
13. Shenoy SM, Shenoy S, Gopal S, Tantry BV, Baliga S, Jain A. Clinicomicrobiological analysis of patients with cholangitis. Indian J Med Microbiol. 2014; 32:157-60.

14. Morris-Stiff GJ, O'Donohue P, Ogunbiyi S, Sheridan WJ. Microbiological assessment of bile during cholecystectomy: is all bile infected? HPB(Oxford). 2007;9:225-8.

15. Kaya M, Beştas R, Bacalan F, Bacaksız F, Arslan EG, Kaplan MA et al. Microbial profile and antibiotic sensitivity pattern in bile cultures from endoscopic retrograde cholangiography patients. World J Gastroenterol 2012;18:3585-9.

16. Suri A, Yasir M, Kapoor M, Aiman A, Kumar A. Prospective study on biliary bacteriology in calcular disease of the gall bladder and the role of common newer antibiotics. The Internet J Surg. 2009;22:2.

17. Bae WK, Moon YS, Kim JH, Lee SH, Kim NH, Kim KA et al. Microbiologic study of the bile culture and antimicrobial susceptibility in patients with biliary tract infection. Korean $\mathrm{J}$ Gastroenterol. 2008;51:248-54.

18. Vaishnavi C, Singh S, Kochhar R, Bhasin DK, Singh G, Singh K Prevalence of Salmonella typhi in bile and stool of patients with biliary disease and those requiring biliary drainage for other purposes. Japanese J Infect Dis. 2005;58:363-5. 